\title{
Registries and collaborative studies for primary ciliary dyskinesia in Europe
}

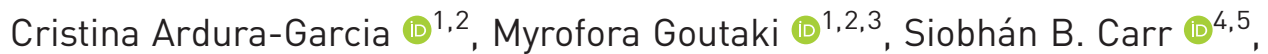 \\ Suzanne Crowley ${ }^{6,7}$, Florian S. Halbeisen $\mathbb{1 0}^{1,8}$, Kim G. Nielsen ${ }^{9,10}$, \\ Petra Pennekamp ${ }^{11,12}$, Johanna Raidt ${ }^{11,12}$, Guillaume Thouvenin (10) ${ }^{13,14,15}$, \\ Panayiotis K. Yiallouros ${ }^{16,17}$, Heymut Omran ${ }^{11,12}$ and Claudia E. Kuehni (1) 1 ,2,3
}

Affiliations: ${ }^{1}$ Institute of Social and Preventive Medicine, University of Bern, Bern, Switzerland. ${ }^{2}$ On behalf of the iPCD Cohort, Bern, Switzerland. ${ }^{3}$ Paediatric Respiratory Medicine, Children's University Hospital of Bern, University of Bern, Bern, Switzerland. "Primary Ciliary Dyskinesia Centre, Dept of Paediatric Respiratory Medicine, Imperial College and Royal Brompton Hospital, London, UK. ${ }^{5}$ On behalf of the English Paediatric PCD Management Service, London, UK. ${ }^{6}$ Paediatric Dept of Allergy and Lung Diseases, Oslo University Hospital, Oslo, Norway. ${ }^{7}$ On behalf of the Norwegian PCD Registry, Oslo, Norway. ${ }^{8}$ On behalf of the Swiss PCD Registry, Bern, Switzerland. ${ }^{9}$ Danish PCD Centre Copenhagen, Paediatric Pulmonary Service, Copenhagen University Hospital, Copenhagen, Denmark. ${ }^{10}$ On behalf of the Danish PCD Registry, Copenhagen, Denmark. ${ }^{11}$ Dept of General Pediatrics, University Hospital Muenster, Muenster, Germany. ${ }^{12}$ On behalf of the International PCD Registry Team, Muenster, Germany. ${ }^{13}$ Service de Pneumologie Pédiatrique, Hôpital Trousseau AP-HP, Sorbonne Université, INSERM, Centre de Recherche Saint-Antoine, CRSA, Paris, France. ${ }^{14}$ Inserm UMR S 933 RaDiCo-PCD, Paris, France. ${ }^{15}$ On behalf of the French RaDiCo-PCD Cohort, Paris, France. ${ }^{16}$ Respiratory Physiology Laboratory, Medical School, University of Cyprus, Nicosia, Cyprus. ${ }^{17}$ On behalf of the Cyprus PCD Registry, Nicosia, Cyprus.

Correspondence: Claudia E. Kuehni, Institute of Social and Preventive Medicine, University of Bern, Mittelstrasse 43, 3012 Bern, Switzerland. E-mail: claudia.kuehnidispm.unibe.ch

ABSTRACT Primary ciliary dyskinesia (PCD) is a rare inherited disease characterised by malfunctioning cilia leading to a heterogeneous clinical phenotype with many organ systems affected. There is a lack of data on clinical presentation, prognosis and effectiveness of treatments, making it mandatory to improve the scientific evidence base.

This article reviews the data resources that are available in Europe for clinical and epidemiological research in PCD, namely established national PCD registries and national cohort studies, plus two large collaborative efforts (the international PCD (iPCD) Cohort and the International PCD Registry), and discusses their strengths, limitations and perspectives.

Denmark, Cyprus, Norway and Switzerland have national population-based registries, while England and France conduct multicentre cohort studies. Based on the data contained in these registries, the prevalence of diagnosed PCD is 3-7 per 100000 in children and 0.2-6 per 100000 in adults. All registries, together with other studies from Europe and beyond, contribute to the iPCD Cohort, a collaborative study including data from over 4000 PCD patients, and to the International PCD Registry, which is part of the ERN (European Reference Network)-LUNG network.

This rich resource of readily available, standardised and contemporaneous data will allow obtaining fast answers to emerging clinical and research questions in PCD.

@ERSpublications

The growing collaborative network of national and international registries and cohort studies of patients with PCD provides an excellent resource for research on this rare disease https://bit.ly/ 3dto75l

Cite this article as: Ardura-Garcia C, Goutaki M, Carr SB, et al. Registries and collaborative studies for primary ciliary dyskinesia in Europe. ERJ Open Res 2020; 6: 00005-2020 [https://doi.org/10.1183/ 23120541.00005-2020].

This article has supplementary material available from openres.ersjournals.com

Received: 6 Jan 2020 | Accepted: 17 March 2020

Copyright CERS 2020. This article is open access and distributed under the terms of the Creative Commons Attribution Non-Commercial Licence 4.0. 


\section{Introduction}

Primary ciliary dyskinesia (PCD) is a rare, genetic, multiorgan disease characterised by dysfunction of motile cilia [1]. The main clinical manifestations are in the upper and lower respiratory tract with chronic rhinosinusitis, recurrent otitis media, chronic wet cough and bronchiectasis, but other organs can be affected [2-5]. About 50\% of patients have situs inversus or situs ambiguous, with manifold variations of left-right asymmetry and a large range of congenital heart defects [6-8]. Most men and some women with PCD are infertile [9-11], and involvement of the central nervous system, olfactory system, inner ear, kidneys and retina has been reported [12]. The clinical phenotype is highly variable [13, 14], partly because of the numerous affected genes that lead to a variety of ultrastructural and functional defects. Prevalence has been estimated as 1 per 10000 or 1 per 20000 , but can be higher in consanguineous societies [15-17]. Although symptoms usually start in the neonatal period, PCD is often diagnosed late and many patients remain undiagnosed [15, 18-20]. Research in PCD has mostly focused on genetic and diagnostic issues; publications on clinical presentation, natural history, prognostic factors and treatments are limited. Publications have typically included few patients from specialised centres, lacked statistical power and precision, and might not be representative for the average patient. Clinical information has come mostly from retrospective chart reviews, is of limited quality and is not comparable across studies [21].

Applied clinical research aims to continuously generate new knowledge to inform patient and clinician decision making. For rare diseases such as PCD, there are many obstacles to clinical research. Adequate sample size is a key limitation, further aggravated by the phenotypic heterogeneity and age-related variability in presentation. While most patients are seen regularly in specialist clinics, with detailed annual reviews, the documentation of these visits in hospital records is not standardised and varies even within institutions. Relevant information is often missing and it remains unclear if missing aspects were not investigated or if results were normal. Lastly, retrospective chart reviews are costly and time consuming. To obtain high-quality clinical data on rare diseases, it is essential that studies are population based, i.e. include all PCD patients living in a region, and provide real-time, standardised prospective data in a shareable format, as a basis for collaborative observational research and as a sampling frame for nested interventional studies. Patient organisations and rare disease specialists have both emphasised the importance of registries [22, 23].

This review describes national and international registries and cohort studies for patients with PCD in Europe, discusses their strengths and limitations, shows how they collaborate, and explains what they have achieved.

\section{National PCD registries or cohort studies in Europe}

This section describes established national registries or cohort studies, which in August 2019 were: 1) population based, i.e. aiming to include every patient living in a defined catchment area, thus being representative and allowing assessment of incidence; 2) based in Europe; 3) long-term initiatives continuously including new patients without a foreseen end; 4) following patients over time to assess disease course and outcomes; and 5) already established and recruiting patients.

For practical reasons we excluded registries from other regions of the world; those which were in the planning stage but not yet functional; single-centre hospital-based studies and projects which were cross-sectional or of limited duration; and those that collected opportunistic clinical data.

We identified the registries presented in discussion with the European PCD research community, which has collaborated closely for years in the PCD Task Forces of the European Respiratory Society (ERS) [15, 24-27], the European Union's Seventh Framework Programme (EU FP7) project BESTCILIA (Better Experimental Screening and Treatment for Primary Ciliary Dyskinesia; www.bestcilia.eu) and the BEAT-PCD (Better Experimental Approaches to Treat PCD; www.beatpcd.org) COST Action network [28, 29]. Details are presented in tables $1-3$. We calculated prevalence of PCD by age group as number of diagnosed PCD patients of a certain age group divided by the population counts of the same age group from national statistics. Figure 1 shows the geographic location of the registries and their contribution to international registries. Denmark, Cyprus, Switzerland and Norway have national population-based registries, while England and France have collaborative studies with longitudinal data from all designated PCD specialist services in the countries. Although not strictly population based, it is assumed that they include most paediatric patients that are diagnosed in the two countries.

\section{Danish PCD Registry}

The Danish PCD Registry, founded in 2006, is hosted by the Danish PCD Centre (Copenhagen, Denmark) and governed by the head of the service. The registry is national and includes all patients diagnosed with PCD in Denmark since 1976. Patients are identified during diagnostic work-up after referral from other 


\section{TABLE 1 Primary ciliary dyskinesia (PCD) registries or cohort studies in Europe with national or near-national coverage:} characteristics and patient identification

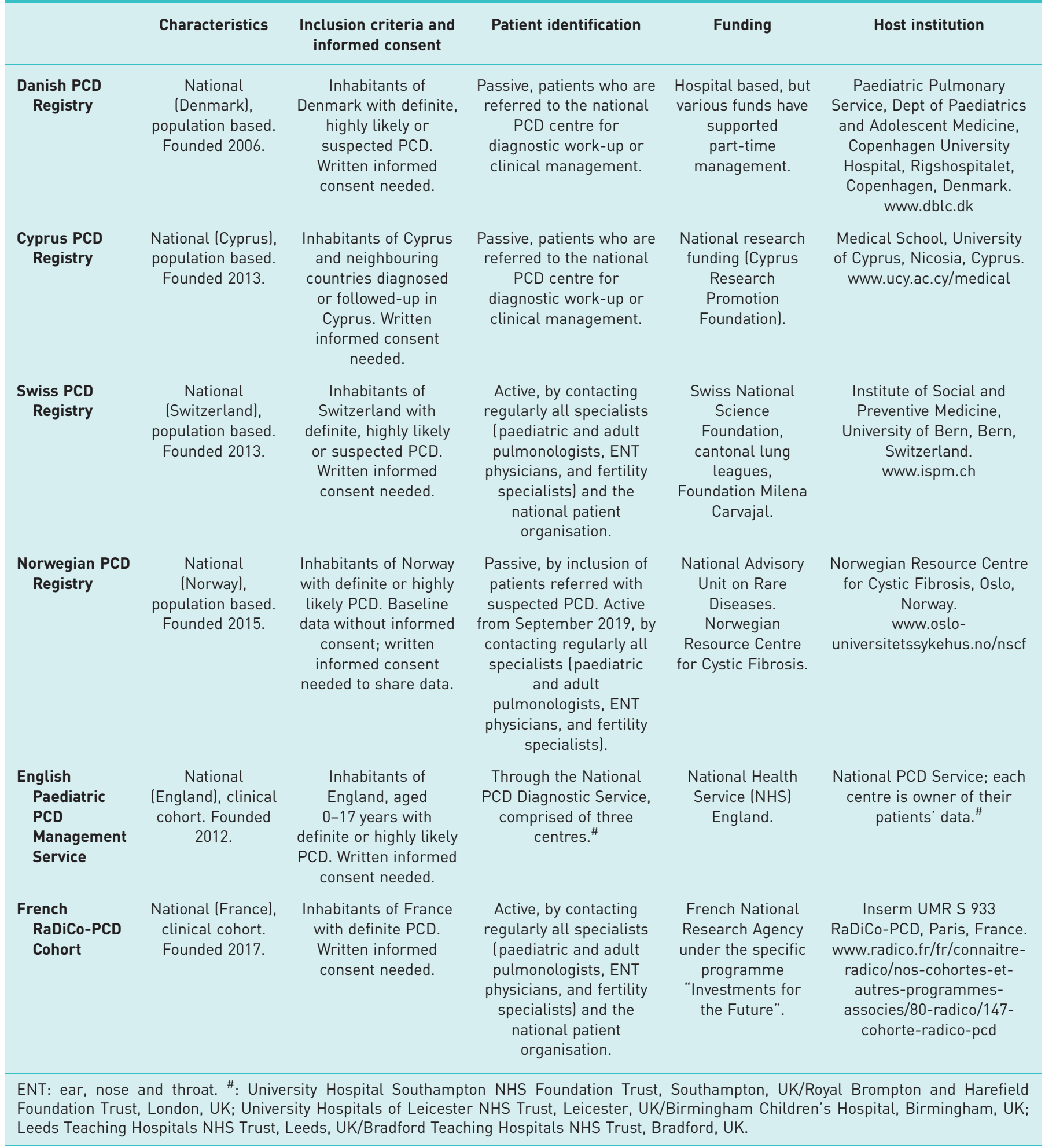

departments across the country (primarily from paediatrics, adult pulmonology, ear, nose and throat (ENT), cardiology, and neonatology) and occasionally from abroad (Sweden and Norway), although the latter are not followed-up after diagnosis and are not included in the counts on prevalence. The registry collects diagnostic information from several years of repeated testing and longitudinal lung function tests (more than 6000 tests). Clinical data are available by searching the EPIC electronic patient records. Data 
TABLE 2 Primary ciliary dyskinesia (PCD) population-based registries in Europe with national or near-national coverage: number of patients diagnosed and registered, and estimated prevalence ${ }^{\#}$

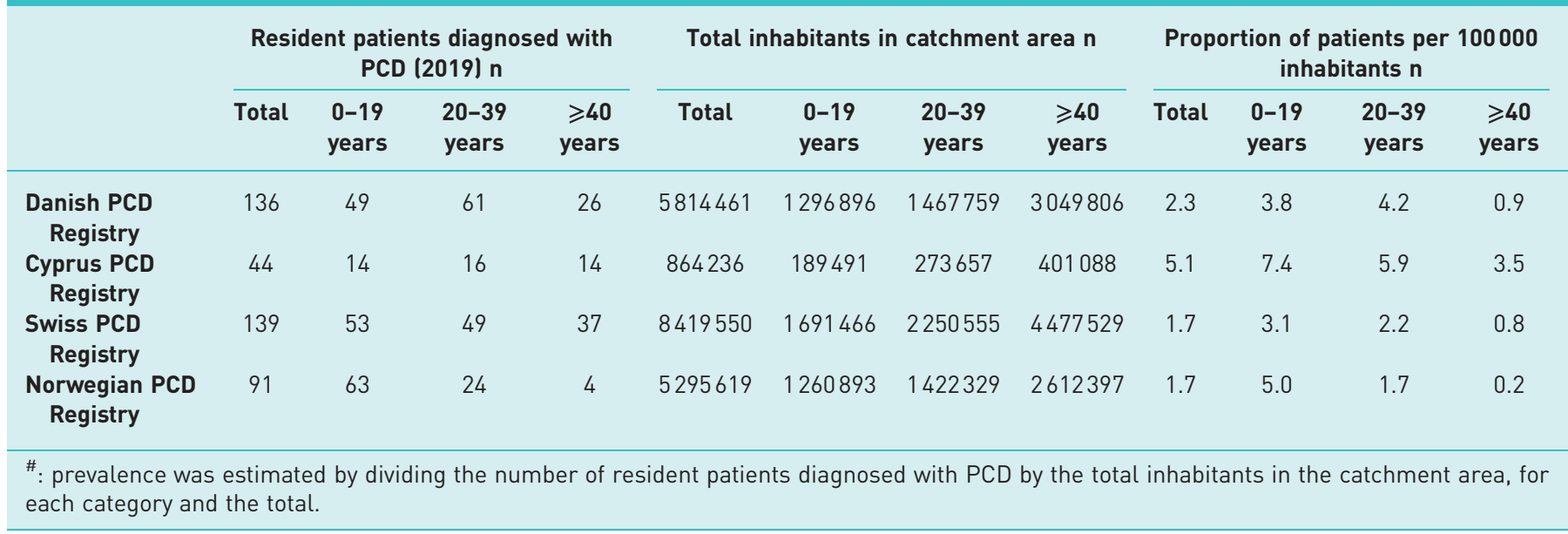

are contributed to the international PCD (iPCD) Cohort [30-35] and the International PCD Registry [36], and have led to many publications [37-49]. Currently, the registry includes 136 alive Danish residents (49 aged $<20$ years, 61 aged $20-39$ years and 26 aged $\geqslant 40$ years). Prevalence of PCD varies from 3.8 per 100000 in $<20$ year olds, 4.2 per 100000 in $20-39$ year olds to 0.9 per 100000 in $\geqslant 40$ year olds.

\section{Cyprus PCD Registry}

The Cyprus PCD Registry, founded in 2013, is hosted by the Medical School of the University of Cyprus (Nicosia, Cyprus). It is population based, and includes all patients with PCD who live in Cyprus or neighbouring countries and have been diagnosed or followed-up in Cyprus. Patients are referred by attending physicians at the single national PCD centre at Archbishop Makarios III Hospital in Nicosia and the data entered in the registry are collected there. Data include demographics, diagnostic information, genotyping and regular clinical follow-up information. Cyprus contributes to the iPCD Cohort [30-35, 50] and the International PCD Registry [36], and has published extensively [51, 52]. Currently, the registry contains data from 51 patients of whom 44 reside in Cyprus (14 aged <20 years, 16 aged 20-39 years and 14 aged $\geqslant 40$ years). Prevalence of diagnosed cases residing in Cyprus is 7.4 per 100000 in $<20$ year olds, 5.9 per 100000 in $20-39$ year olds and 3.5 per 100000 in $\geqslant 40$ year olds.

\section{Swiss PCD Registry}

The Swiss PCD Registry, founded in 2013, is hosted by the University of Bern (Bern, Switzerland) and governed by a national advisory board including all physicians who register patients [53]. It is population based, aiming to include all patients with PCD living in Switzerland, and is registered at ClinicalTrials.gov with identifier number NCT03606200. Patients are identified actively by regular contacts with physicians of all specialities likely to see PCD patients (paediatric and adult pulmonologists, ENT physicians, fertility specialists, cardiologists and neonatologists). The registry collects diagnostic data, clinical data from encounter records, linked routine data (from birth, mortality, census and hospital statistics) and patient-reported information from questionnaire surveys. It has contributed to several publications of the iPCD Cohort [30-35, 50] and the International PCD Registry [36]. Currently, the registry includes 139 patients (53 aged $<20$ years, 49 aged $20-39$ years and 37 aged $\geqslant 40$ years). Prevalence of diagnosed cases varies from 3.1 per 100000 in <20 year olds, 2.2 per 100000 in $20-39$ year olds to 0.8 per 100000 in $\geqslant 40$ year olds.

\section{Norwegian PCD Registry}

The Norwegian PCD Registry, founded in 2015, is hosted by the Norwegian Resource Centre for Cystic Fibrosis (Oslo, Norway) and governed by an advisory board that includes physicians who contribute patients. It is population based, aiming to include all patients with PCD living in Norway. Patients are identified following referral for confirmation of diagnosis and there is planned active recruitment from late 2019 by direct contact with physicians of all specialities likely to see PCD patients. Data include diagnostic information and regular follow-up data. Registry data can be linked to routine national statistical data and to Norwegian neonatal and congenital heart disease registries. Data are contributed to the iPCD Cohort $[30-34,50]$ and the International PCD Registry. Currently, the registry includes 91 patients (63 aged 
TABLE 3 Primary ciliary dyskinesia (PCD) registries or cohort studies in Europe with national or near-national coverage: data collection and contribution

$\begin{array}{cccccc}\text { Danish PCD } & \text { Cyprus PCD } & \text { Swiss PCD } & \text { Norwegian } & \text { English Paediatric } & \text { French } \\ \text { Registry } & \text { Registry } & \text { Registry } & \text { PCD Registry } & \text { PCD Management } & \text { RaDiCo-PCD } \\ & & & & \text { Service } & \text { Cohort }\end{array}$

\section{Collection of baseline data}

Extraction from patient records

Results of diagnostic tests from national PCD service

Standardised form (FOLLOW-PCD) completed prospectively during visits

Collection of follow-up data

Periodic data collection from patient records

Annual or 3-monthly review assessments

Standardised follow-up form

Questionnaire to patients

Type of data collected

Diagnostic information

Clinical examinations

Lung function tests

Audiology

Imaging

Microbiological results

Blood tests le.g. liver function, immune profiling)

Medical and surgical treatments

Patient-reported data

Annual QOL-PCD questionnaire

National patient surveys

Linkage with routine statistics

National mortality registry

National birth/neonatal registry

Census data

Hospital episode statistics

\section{Contributes to}

iPCD Cohort

International PCD Registry

National Rare Disease Registry (FOLLOW-PCD)

Congenital heart disease registry

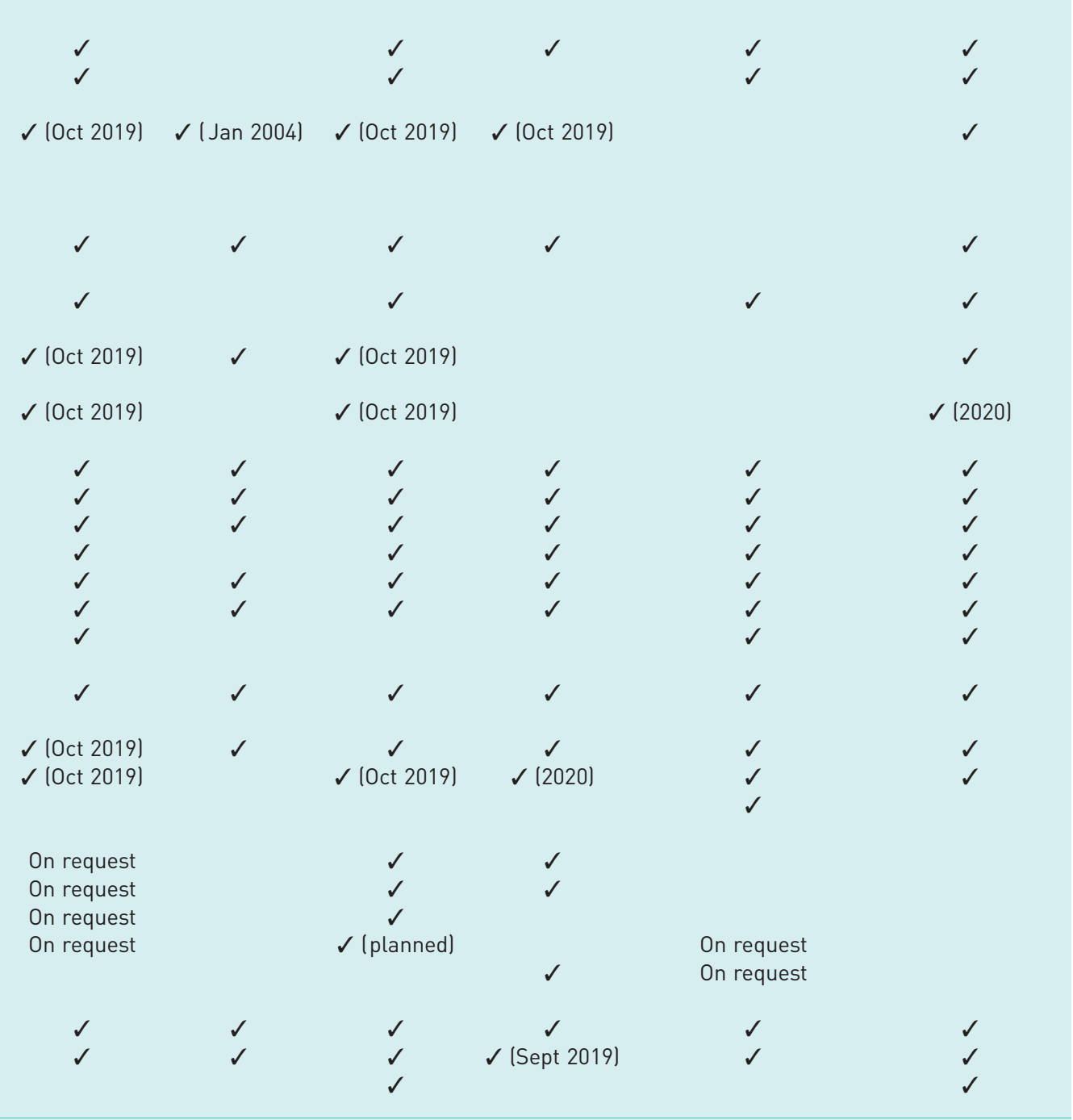

QOL: quality of life.
$<20$ years, 24 aged $20-39$ years and four aged $\geqslant 40$ years). Prevalence of diagnosed cases varies from 5.0 per 100000 in $<20$ year olds, 1.7 per 100000 in $20-39$ year olds to 0.2 per 100000 in $\geqslant 40$ year olds.

\section{English Paediatric PCD Management Service}

The English Paediatric PCD Management Service was commissioned by the National Health Service (NHS) England in 2012 to complement the existing diagnostic service [54]. Patients are identified by the National PCD Diagnostic Service, established in 2006 [55]. Children with a confirmed or "highly likely" diagnosis [26] following testing receive annual review assessments in one of four PCD management centres (Leeds, Leicester, London or Southampton) from a specialist PCD multidisciplinary team following a standardised service specification. The service collects diagnostic information, annual clinical assessments (symptoms and examinations), investigations (microbiology, spirometry, audiology, etc.) and information on treatments, contributes to the iPCD Cohort $[30-35,50]$ and the International PCD Registry [36], and has published extensively [19, 56-74]. Currently, the service includes 396 children, of a median age of 9.8 years, $53 \%$ males, the majority being white British (37\%) or Pakistani/British Pakistani (36\%). A parallel nationally commissioned adult service will start in 2020. Prevalence of diagnosed cases in the paediatric population is 2.7 per 100000 . 


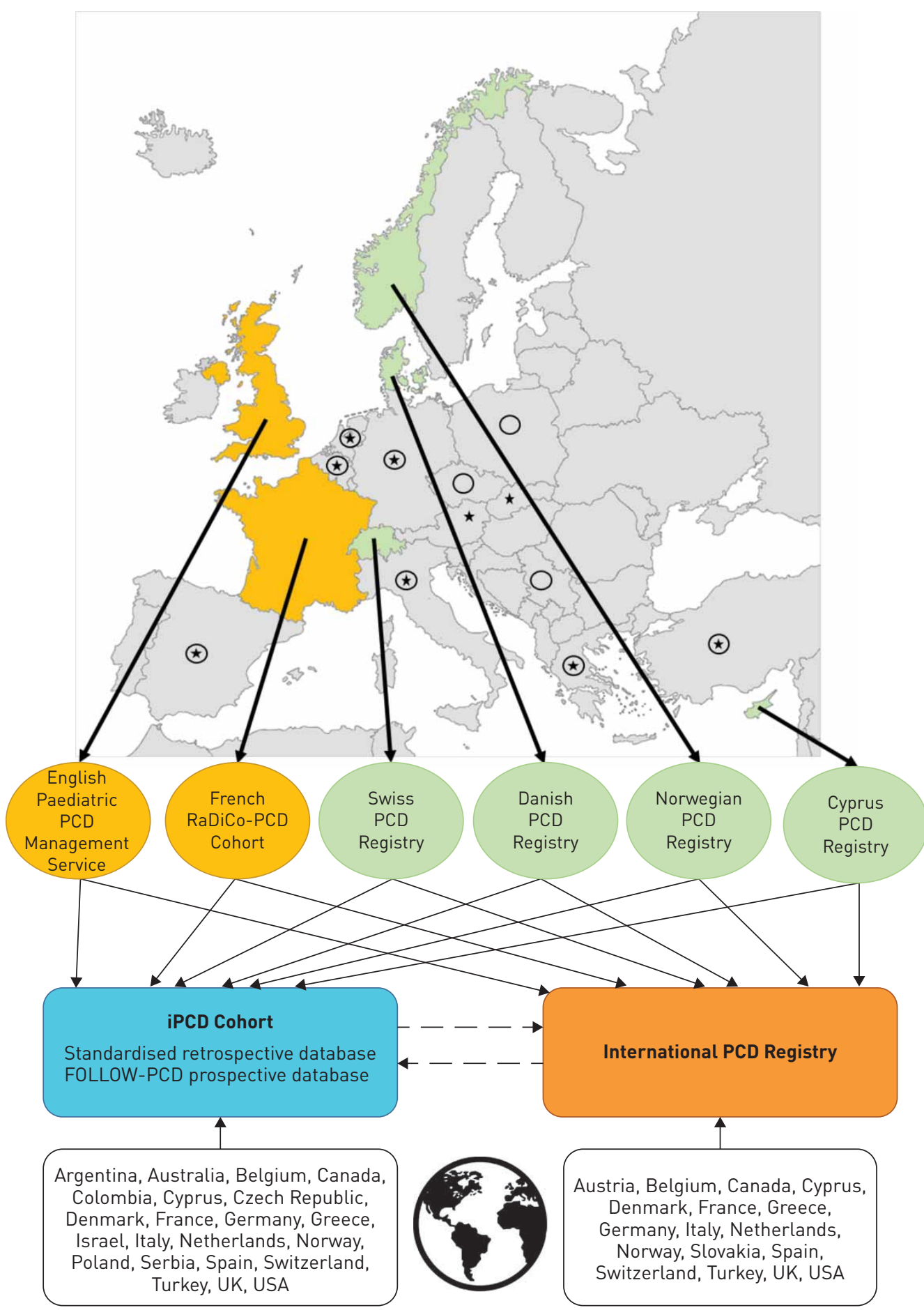

FIGURE 1 Countries in Europe with established national population-based registries or multicentre cohort studies and data flow between them and the international primary ciliary dyskinesia (iPCD) Cohort and the International PCD Registry. Yellow ovals: clinical cohorts; green ovals: population-based registries; black continuous lines: data contribution; black dashed lines: data sharing and collaborations; black stars: other European countries that contribute data to the International PCD Registry; black circles: other European countries that contribute data to the iPCD Cohort.

\section{French RaDiCo-PCD Cohort}

The French RaDiCo-PCD Cohort was founded in 2017 with the Research National Agency as a sponsor, under the authority of the French Minister of Higher Education, Research and Innovation, and sustained by the French National Rare Lung Disease Network. The cohort is hosted at the French National Institute of Health and Medical Research (Institut National de la Santé et de la Recherche Médicale, Inserm, Paris, 
France) (UMR S $933 \mathrm{RaDiCo}$ ), and is governed by a multidisciplinary board of physicians, researchers, patients and experts in organisation and information systems. The main goal of RaDiCo-PCD is to identify factors that predict disease severity in paediatric and adult PCD patients with well-established diagnosis, followed in certified Rare Lung Disease centres [21, 75, 76]. It collects diagnostic information and data from regular follow-ups. RaDiCo-PCD contributes to the iPCD Cohort [30-32, 34, 35], and has reported on sino-nasal disease and fertility $[9,77,78]$. Currently, RaDiCo-PCD includes 185 patients $(80$ aged $<20$ years, 66 aged $20-39$ years and 39 aged $\geqslant 40$ years). Prevalence of diagnosed cases varies from 0.6 per 100000 in <20 year olds, 0.4 per 100000 in $20-39$ year olds to 0.1 per 100000 in $\geqslant 40$ year olds, as it is a very new cohort, but increases rapidly with several new cases diagnosed or recruited each year.

\section{Others}

Several other countries in Europe have cross-sectional or longitudinal datasets on PCD patients treated in specialised centres, without having a national registry or cohort study. These include Belgium, Italy and the Netherlands. Most of these contribute either to the iPCD Cohort or the International PCD Registry, or both. As they are not population based, however, data cannot be used to estimate incidence or prevalence and it is unclear if they are representative for all patients in the respective country.

\section{Collaborative studies: the iPCD Cohort and the International PCD Registry}

The two large collaborative studies were both set up in BESTCILIA (2013-2016) in the EU FP7. Work package 1 of BESTCILIA assembled available datasets on PCD in a retrospective international cohort study, the iPCD Cohort [31], and work package 2 set up a prospective International PCD Registry [36]. Both datasets were further expanded through the COST Action BEAT-PCD into large, still growing datasets $[25,28,29]$. They have different strengths and limitations, and the datasets are overlapping because many centres contribute to both initiatives (table 4 ).

\section{The iPCD Cohort}

The iPCD Cohort is hosted by the University of Bern and governed by an international advisory board including representatives from all contributing centres [31]. It aims to answer questions on clinical presentation, severity, prognosis and treatments in patients with PCD. The iPCD Cohort was originally set up as a retrospective international cohort, combining existing datasets on patients with PCD, but now also collects prospective follow-up information. Since 2019 the iPCD Cohort has been linked with FOLLOW-PCD, the standardised clinical form for documenting patient encounters in PCD clinics [79].

Eligible datasets and centres were initially identified by a systematic literature search for studies describing clinical manifestations in patients with PCD [80], through personal contacts from the ERS PCD Task Force (2006-2009) $[15,27]$ and from the BEAT-PCD network. New centres that wish to contribute data can always join.

The iPCD Cohort collects basic patient information, results of diagnostic tests, baseline clinical history, and data on growth, lung function, microbiology, imaging, clinical manifestations and treatments. Data is contributed on a standard format that was developed in 2013 , in discussion with all contributors. This was based on the data available in the local datasets and therefore not very detailed. With the standardised PCD follow-up form (FOLLOW-PCD), the iPCD Cohort now has the possibility to include detailed physician-reported prospective data and patient-reported information on symptoms, health behaviour and environmental exposures.

Currently, the iPCD Cohort includes data from 3824 patients from 22 countries in Europe, the Middle-East, North and South America, and Australia. Each contributor ensures that the delivered data are pseudo-anonymised and in accordance with the national/local data protection laws. Local principle investigators are responsible for obtaining local ethical approval and patient informed consent, and the process is supported by the iPCD Cohort management team. Identifying information linking the data to the patients is kept by the centre and not included in the iPCD Cohort. Agreements for data delivery and publication leave all rights with the data contributors. For each planned analysis, eligible data are validated, cleaned and standardised. So far, the iPCD Cohort has published data on growth, nutrition, lung function and PCD diagnostics $[30-35,50]$.

Centres that wish to participate to the iPCD Cohort can contact pcd@ispm.unibe.ch to receive detailed instructions on how to proceed. Each contributor signs a data delivery agreement and receives a password to access the online software REDCap to enter data directly [81]. Alternatively, data that are already in the standard format can be contributed as a data file, e.g. as an export from a national registry or the International PCD Registry. Centres keep constant access to their datasets and can export them in various formats for local analyses. Researchers wanting to use the iPCD Cohort dataset can propose a topic and a concept sheet describing the planned analyses and publication. Participating centres can always decide if 
TABLE 4 International PCD (iPCD) Cohort and International PCD Registry: characteristics and methods

\section{iPCD Cohort}

Cohort study, collaborative dataset

2013

Year founded

Inclusion criteria

National, collaborative or single-centre datasets of patients (any age) diagnosed and treated for PCD

National legal and ethical requirements met by participating centre

Both cross-sectional and longitudinal datasets accepted (longitudinal encouraged)

Patients with probable or confirmed PCD diagnosis who are treated as PCD in the participating centres, ranging from clinical presentation consistent with PCD to diagnosis established with several PCD diagnostic tests based on the European Respiratory Society diagnostic recommendations

Written informed consent needed for prospective data, depending on national laws

Patients (2019) n 3824

Participating Argentina, Australia, Belgium, Canada, Colombia, Cyprus, countries Czech Republic, Denmark, France, Germany, Greece, Israel, Italy, Netherlands, Norway, Poland, Serbia, Spain, Switzerland, Turkey, UK, USA

\section{Prospective/ retrospective}

Type of data

ClinicalTrials.gov identifier

Database

REDCap database

Easy data import and export in several formats

Each collaborator has access to own data

\section{Funding}

Host institution

Institute of Social and Preventive Medicine, University of Bern, Bern, Switzerland

www.ispm.unibe.ch

\section{To contribute}

Contact: pcddispm.unibe.ch

\section{International PCD Registry}

Registry

2014

National, collaborative or single-centre datasets of patients (any age) diagnosed and treated for PCD

National legal and ethical requirements met by participating centre

Patients with a confirmed PCD diagnosis fulfilling the following diagnostic criteria: clinical presentation consistent with PCD and consistent findings specific for PCD by at least two methods (high-frequency video microscopy analysis, transmission electron microscopy, immunofluorescence microscopy and low nasal nitric oxide concentration/production or biallelic diseasecausing mutations by genotypingl

Entering individual patients requires written informed consent by the patient or his/her legal guardian(s)

920

Austria, Belgium, Canada, Cyprus, Denmark, France, Greece, Germany, Italy, Netherlands, Slovakia, Spain, Switzerland, Turkey, UK, USA

Prospective (and retrospective)

Pseudo-anonymised

NCT02419365

Internet-based data entry system; will be transferred to REDCap October 2019

Easy data import and export in several formats

Each collaborator has access to own data

Basic data set is monitored for completeness, further items are currently checked manually for plausibility by experts

EU-funded BESTCILIA project (FP7/2007-2013) under grant agreement 305404, Eva Luise Köhler Research Award, Care-for-Rare Science Award, REGISTRY WAREHOUSE (Horizon 2020, grant agreement 777295), DFG CRU326 (OM6/11) and institutional funding

University Hospital Muenster, Westphalian University of Muenster, Muenster, Germany www.ukm.de www.pcdregistry.eu

Contact: PCDRegistry.eudukmuenster.de data

they want to contribute with their data to a planned analysis and be part of a particular project. Nested studies using part of the iPCD dataset and collecting additional data are encouraged with methodological input and support by the iPCD Cohort team. Such data will be added to the iPCD Cohort to enrich it after each project. 
The International PCD Registry

The International PCD Registry, launched in 2014, is governed by a steering committee of the University Children's Hospital Muenster (Muenster, Germany) [36]. Since 2019 it has been hosted in the Institute of Medical Informatics at the University of Muenster. Lately, it has been expanded through the REGISTRY WAREHOUSE project of the ERN (European Reference Network)-LUNG (https://ern-lung.eu/ern-lung-rdregistry-data-warehouse). It is now registered with the European Rare Disease Registries Infrastructure (https://eu-rd-platform.jrc.ec.europa.eu/erdri_en).

The International PCD Registry is designed as a prospective registry, but it is also possible to include retrospective data. It aims to evaluate clinical presentation, disease course, treatments, outcomes and time trends, and facilitates recruitment of patients for research studies. Participating centres contribute data continuously and additional centres can join.

Eligible datasets and collaborating centres were initially identified through the BESTCILIA project and the BEAT-PCD network. For ERN-LUNG PCD CORE members and associated partners, participation in the International PCD Registry is mandatory in order to fulfil EU requirements.

The International PCD Registry collects data on diagnostics (high-speed videomicroscopy, transmission electron microscopy, nasal nitrite oxide, immunofluorescence microscopy and genotype), age and health status at diagnosis, family history, clinical manifestations, associated malformations/diseases, progression of the disease, exacerbations, microbiology, lung function, radiological findings, therapeutic measures (inhalation, antibiotics, oxygen, ventilation, ENT and lung surgery), date and cause of death, fertility, and quality of life using PCD-specific instruments [57]. For participating centres without access to genetic testing, this is offered within the framework of research projects.

Currently, the International PCD Registry includes 920 patients from 23 centres in 16 countries in Europe, the Middle-East, Canada and North America. After written informed consent by the patient or his/her legal guardian(s), individual patient data can be entered directly. The platform can also integrate data from local databases, avoiding the need to enter data twice. Informed consent remains with the referring centre to preserve pseudo-anonymity. Withdrawal of authorisation is possible at any time and all information will then be destroyed. Healthcare providers can include patients after they obtained local ethical approval. Publication of the data from individual centres is only possible when the participating centre consents.

Centres that wish to participate in the International PCD Registry need to meet all necessary legal and ethical requirements. This process is guided by the registry management team. Informed consent forms and protocols for data collection have been approved by the Ethical Review Committees of the Medical Council Westfalen-Lippe and the Medical Faculty of the Westphalian Wilhelms University Muenster.

Each referring centre only has access to its own patients' data. Only the administration team has insight into data of all patients in an anonymised way. Information linking subject identifier (ID) with personal data identifying the individual is kept only in the referring centre and is not collected in the registry. To get access to registry data or recruit participants for research studies the registry steering committee comprising members of the ERN-LUNG PCD Core group has to be contacted via PCDRegistry.eu@ukmuenster.de. Planned investigations should be introduced as a short concept sheet, which will be evaluated by the committee for feasibility and scientific impact. All referring centres will be asked for approval for utilisation of patient data. For further details, refer to www.pcdregistry.eu or contact PCDRegistry.eu@ukmuenster.de.

\section{Differences between the IPCD Cohort and the International PCD Registry}

Despite their similarities, these two independent initiatives present some important differences. The iPCD Cohort's inclusion criteria are broader than those of the International PCD Registry, including also patients with probable or clinical diagnosis of PCD, which results in a larger number of patients included, but also in less detailed and standardised data. However, the iPCD Cohort is from its set-up more research oriented, resulting in many publications. In contrast to the International PCD Registry, patient data are not always included at regular time intervals, but relevant data are added at irregular intervals, on the occasion of planned analyses. Lastly, by including more detailed data on diagnostic evaluations, the International PCD Registry can be used to identify eligible patients for randomised clinical trials studying specific diagnostic groups.

\section{Data exchange and collaboration}

Figure 1 illustrates data flows between national registries, the iPCD Cohort and the International PCD Registry. All national registries and clinical cohorts described in this review contribute data to the iPCD Cohort and the International PCD Registry. Some national registries have started to use the standard clinical form FOLLOW-PCD to collect detailed clinical encounter data. Interested researchers may request 
data from the iPCD Cohort and the International PCD Registry to answer specific questions. Each patient has a unique iPCD-ID number for the iPCD Cohort as well as the original dataset ID. The iPCD Cohort and International PCD Registry are independent of each other, but collaborate. As the datasets for the iPCD Cohort and the International PCD Registry were developed together, many variables are common to both datasets and have the same format, permitting easy data transfer between the two databases. In addition, several national registries (e.g. Swiss and Norwegian) have the same format, thus allowing direct transfer of local data to the iPCD Cohort and the International PCD Registry for specific analyses.

\section{Important findings}

National and international studies have helped to characterise disease presentation and course, and to provide first evidence to guide patient diagnosis and care. Several studies focused on lung function. An iPCD study including 991 patients found that PCD affects lung function from early in life, comparable to cystic fibrosis (CF) [32]. Gas exchange [78] and cardiopulmonary fitness seem to be reduced in patients with PCD, similarly as in CF [39, 49]. Adult patients from France had considerable variability in lung impairment, usually moderate but more severe in women and patients with chronic infection with Pseudomonas aeruginosa [21]. Patients from Cyprus diagnosed late in adulthood had advanced lung disease and prior lobectomy was a poor prognostic factor for the disease course [51]. Longitudinal studies in children and young adults reported stable spirometry but slight increases in the lung clearance index in children and young adults with PCD $[40,48]$. There is increasing evidence that lung function is associated with the type of ultrastructural defect $[32,74]$. Mannose-binding leptin (MBL) deficiency was associated with steeper lung function decline in Danish PCD patients, suggesting that the MBL phenotype might be a disease modifier in PCD [82].

Growth and nutrition in PCD patients can be impaired from early childhood, and are strongly associated with lung function [30]. A study in the UK suggests that childhood monitoring of nutritional status and vitamin D levels could help to identify children at risk who would require nutritional support [63]. Data on upper airways from France suggest that adult patients commonly suffer from rhinosinusitis but less frequently from otitis media with effusion. The latter was associated with a worse lung function [77]. Studies from the Danish registry found simultaneous sinus and lung infections with the same bacterium [47], and improvements in chronic rhinosinusitis symptoms and lung function after sinus surgery [46]. Only few studies focused on non-respiratory manifestations such as infertility, situs defects and congenital heart disease $[9,58]$.

\section{Discussion}

This article gives an overview of the European registries and collaborative studies for PCD. While a decade ago clinical research in PCD relied on small cross-sectional studies and case series, with information assessed at one time-point or from retrospective charts reviews, we now have large population-based registries and collaborative studies in Europe. This allows us to tackle open questions using standardised representative and prospective datasets.

Population-based national registries aim to include every patient with PCD in a country, allowing us to assess incidence and prevalence, and to describe the full range of clinical presentation and prognosis. The summaries presented review the current state of the registries and problems they have to overcome. Registries are easier to lead in countries with centralised care, such as Cyprus or Denmark with only one national PCD centre. In such a system, every resident diagnosed with PCD has a record in one of the dedicated clinics and a clinical registry is automatically completed. In countries with decentralised care, such as Switzerland, patients are seen in numerous clinics and private practices, and registries must actively search for new patients by regularly contacting all medical specialists likely to see PCD patients (pulmonologists, ENT physicians, cardiologists, neonatologists and fertility specialists) and patient networks, which leads to high costs. Other countries with decentralised care, such as Norway, follow-up all known PCD patients at least annually in one centralised clinic using a shared-care strategy.

Prevalence data (table 2) suggest, however, that only a fraction of adult patients are diagnosed and thus included in registries [53]. For children and adolescents aged 0-19 years, estimated prevalence was 7 per 100000 in Cyprus and 3-5 per 100000 in Norway, Denmark and Switzerland. Considering late diagnosis in children with less severe presentation, these data support the estimate that PCD affects about 1 per 10000 people. Prevalence of PCD in adults is much lower in all registries, around 0.2-2 per 100000 in Norway and Switzerland, and up to 4-6 per 100000 in Cyprus and Denmark, where single national PCD centres exist. Although life expectancy in PCD might be slightly reduced, this does not explain the prevalence gap between children and adults [15]. The likely explanation is a considerable underdiagnosis of PCD in adults that are treated under wide diagnostic "umbrellas", e.g. non-CF bronchiectasis, and missing registration. The lack of funding to reimburse centres or doctors for data entry into national 
registries (from where international collaborations obtain their data too) may also reduce data collection completeness, especially in adults. This does not only lead to low precision of results for adult patients, e.g. on lung function [30,32,36], but also to biased and potentially wrong results. It is impossible to know whether registered adult patients represent the average patient, or rather the upper or lower end of the severity spectrum. Although numbers are rising and there are initiatives such as the ERS Task Force registry EMBARC (www.bronchiectasis.eu) and the UK Bronch-UK (www.bronch.ac.uk), adult pulmonologists remain massively under-represented in PCD task forces and networks. This will hopefully change with the establishment of PCD excellence centres for adults in the UK and elsewhere.

Two large collaborative studies, the iPCD Cohort and the International PCD Registry, combine data from registries and single-centre studies from Europe and beyond into large research datasets with sufficient statistical power and precision for different types of analyses. Both initiatives have key strengths that provide opportunities for different types of research. The iPCD Cohort includes more patients (nearly 4000), as it also includes older datasets. The International PCD Registry is smaller, but growing, and has potential as it now receives continuous support from the EU in the ERN-LUNG network. It contains detailed data on results from diagnostic evaluations, particularly genetics, and will allow genotypephenotype association studies to be performed in the future. Both the iPCD Cohort and the International PCD Registry collect a minimal core dataset of clinical information. This can be overcome by focused collection of additional data in nested studies, which can be fed back to the original dataset to enrich it further. This approach has worked well for the iPCD Cohort where some national registries register data in iPCD continuously, while others contribute tailored datasets for specific analyses and publications. The introduction of FOLLOW-PCD, a standardised form for collecting data during routine care, will greatly improve the richness of the dataset.

Outside Europe, there are other established or emerging studies on patients with PCD. An important resource is the PCD Foundation Clinical and Research Centers Network in North America, which has recently set up the PCD Foundation Registry collecting health and demographic data of PCD patients who are followed in the participating centres (https://pcdfoundation.org/registry). In Australia, a clinical care and research network has been developed between Sydney and Melbourne [83, 84], and Japan has recently conducted a nationwide survey of PCD patients as a first step towards the development of a national registry [85].

Similar efforts in other rare diseases have paved the way for significant advances in the epidemiological and clinical knowledge on rare diseases. Examples in pulmonary medicine include registries for CF [86, 87], non-CF bronchiectasis [88], interstitial lung disease [89], $\alpha_{1}$-antitrypsin deficiency [90] and pulmonary hypertension [91]. ERN-LUNG (https://ern-lung.eu), the Network of European Healthcare Providers dedicated to care and research for patients with rare respiratory diseases, will provide further means and platforms to coordinate and standardise care for rare lung diseases, and provides funds for the International PCD Registry.

The most advanced and best-know examples of registries for rare diseases are cancer registries [92]. A systematic registration of cancer (many of which are rare, including all childhood cancers) was set up in the 1950s by pioneer countries (e.g. the Nordic countries and the UK) and has expanded since then; coverage is now almost universal. Cancer registries include large numbers of patients and thus usually collect a limited dataset. Completeness of case registration is usually high (over 95\% of affected patients), and therefore provides reliable data on incidence and survival. Time trends and regional differences in incidence allow us to investigate potential causes of cancer, while survival differences can be used to investigate differences in healthcare between countries. Cancer registries also serve as sampling frames to recruit patients into randomised clinical studies, which compare new treatment options with the current standards of care [93]. The routine of including a large number of patients into clinical trials led to continuous improvements in childhood cancer survival, with a cure rate of over $85 \%$ [94, 95]. Such rapid improvements in outcomes have hardly been reached in other rare diseases, where patients are not cared for centrally, not reported to registries and not routinely included in trials. Exact residential addresses in cancer registries have allowed investigation into environmental risk factors for cancer incidence comparing geographically and varying environmental exposures (such as ionising radiation or air pollution) [96, 97]. Lastly, cancer registries have served as baselines for lifelong follow-up studies on long-term morbidity, mortality and quality of life, permitting us to study long-term toxicities of specific treatment modalities, and to adapt treatment of new patients to optimise cure rates while minimising long-term side-effects [98].

Funding is a continuous problem for rare disease registries, as money is scarce and patients are scattered. The longer history and the population-based setting of cancer registries allowed them to develop efficient and cheap ways of data collection. While most pulmonary registries still rely on manual data entry by physicians and active follow-up of individual patients for vital status, cancer registries have developed 
more efficient routines. Importantly, they often operate without collecting informed consent, which is time consuming and expensive, but based on a legislation that provides for ample information of patients and the right to opt out, rather than to opt in. Data collection is as automatic as possible, with pathology laboratory and hospital statistics automatically delivering cancer diagnoses to registries, and survival and causes of death being ascertained via data linkage with national mortality records. This leads to cheaper, more complete and more sustainable ways of data collection, which could also easily be adopted by other registries. Lastly, the recent developments facilitating the automated use of the data contained in hospital information systems should be used for rare diseases $[99,100]$. However, this is dependent on national legislation and needs strong protection data measures, as many rare diseases are genetic.

In summary, while a decade ago clinical data on patients with PCD were available only from small cross-sectional studies of selected patients, we now have a valuable and fast-expanding network of national population-based registries and collaborative studies. The registries may be further improved if they are standardised, with more efficient data collection and data sharing, improve data quality, use information that is routinely collected during healthcare and are able to analyse the data quickly, ideally in real-time. These datasets have a huge potential to help answer many open questions in the clinical epidemiology of PCD and serve to provide continuous evidence-based improvements in care and quality of life of affected patients.

Acknowledgements: We would like to thank the following collaborators for their contribution in setting up and maintaining the PCD registries and cohort studies. Cyprus PCD Registry: Panayiotis Kouis, Respiratory Physiology Laboratory, Medical School, University of Cyprus, Nicosia, Cyprus. English Paediatric PCD Management Service: Jane Lucas, Claire Hogg, Chris O'Callaghan, Woolf Walker, Siobhan B. Carr, Anton Page, Patricia Goggin, Claire Jackson, Regan Doughty, Janice Coles, James Thompson, Bruna Rubbo, Corine Driessens, Samantha Packham, Amelia Shoemark, Sarah Ollosson, Charlotte Richardson, Farheen Daudvohra, Ranjit Rai, Paul Griffen, Andrew Rogers, Robert Hirst, Andrew Rutman. Philip Chetcuti, Manjith Narayanan, Priti Kenia, Eduardo Moya, Amanda Harris, Amanda Friend, Victoria Keenan, Hannah Wilkins, Hasnaa Ismail-Koch, Andrea Burgess, Gemma Marsh, Laura Baynton, Michael Leshen, Nicola Collins, Peter Rea, Ann-Louise McDermott, Amanda Parsons, Rajinder Kang, Nicola Lloyd, Alison Truscott and Lynne Schofield. The following have been instrumental in setting up and maintaining the registries and cohort studies. PCD Family Support Group (chair: Fiona Copeland) of NIHR Southampton Biomedical Research Centre (Respiratory theme and Data Science theme). French PCD cohort: French RaDiCo-PCD Cohort (coordination: S. Amselem; scientific and operational direction: S. Gueguen) and the French National Rare Lung Disease Network (RespiFil, RespiRare coordination: A. Clement). iPCD Cohort: Myrofora Goutaki, Claudia E. Kuehni, Florian Halbeisen, Eugenie Collaud and Cristina Ardura-Garcia. We thank all the patients with PCD in the iPCD Cohort and their families, and especially the PCD patient organisations for their close collaboration. We also thank all the researchers in the participating centres who were involved in data collection and data entry, and worked closely with us through the whole process of participating to the iPCD Cohort. The International PCD Registry Team: Heymut Omran, Martin Dugas, Simone Helms, Petra Pennekamp, Johanna Raidt and Sarah Riepenhausen. We thank all the patients with PCD and their families and the PCD patient organisations for their close collaboration and support of national and international registries and cohort studies. We also thank all the researchers of the participating centres who are or were involved in data collection and data entry. The following have been instrumental in setting up and maintain the registry: Claudius Werner, Simone Helms, Maria Teekat, Nadja Hackmann, Martin Dugas, Sarah Riepenhausen, Martin Lablans and Frank Ückert. The national and international centres that contribute data to the iPCD Cohort and/or the International PCD Registry are shown in supplementary table S1.

Conflict of interest: C. Ardura-Garcia has nothing to disclose. M. Goutaki has nothing to disclose. S.B. Carr reports service on an advisory board for, a lecture fee from, travel support from and service on a steering committee for Vertex Pharmaceuticals; service on an advisory board for Profile Pharmaceuticals; lecture fees from Teva Pharmaceuticals; service on an advisory board for, and travel and accommodation support from Chiesi Pharmaceuticals, all outside the submitted work. S. Crowley has nothing to disclose. F.S. Halbeisen has nothing to disclose. K.G. Nielsen has nothing to disclose. P. Pennekamp has nothing to disclose. J. Raidt has nothing to disclose. G. Thouvenin has nothing to disclose. P.K. Yiallouros has nothing to disclose. H. Omran has nothing to disclose. C.E. Kuehni has nothing to disclose.

Support statement: This study was supported by Swiss National Science Foundation (320030_173044 to C.E. Kuehni). The Cyprus PCD Registry has received funding from the Cyprus Research Promotion Foundation grants IPE/KY/ ROU-0311/11 and IPE/PENEK/ENTAX/0506-24. The National PCD Service England is commissioned and funded by NHS England. The French RaDiCo-PCD Cohort is funded by the French National Research Agency under the specific programme "Investments for the Future", cohort grant agreement ANR-10-COHO-0003 (https://anr.fr/en). The Norwegian PCD Registry is funded by the National Advisory Unit on Rare Diseases and the Norwegian Resource Centre for Cystic Fibrosis. The development of the iPCD Cohort has been funded from the European Union's Seventh Framework Programme (EU FP7) under grant agreement 305404 BESTCILIA (Better Experimental Screening and Treatment for Primary Ciliary Dyskinesia). It is supported by the Swiss National Science Foundation SNSF 320030_173044 and the Pro-Kartagener Foundation Milena Karvajal. The International PCD Registry is supported by REGISTRY WAREHOUSE (Horizon 2020, grant agreement 777295) and DFG CRU326 (OM6/11). The development has been funded from EU FP7 under grant agreement 305404 BESTCILIA. Funding continues within several projects including REGISTRY WAREHOUSE (Horizon 2020, grant agreement 777295) and is supported by funding through Scientific Awards to H. Omran (Eva Luise Köhler Research Award and Care-for-Rare Science Award), the DFG Clinical Research Unit (CRU) 326 (OM6/11) and institutional funding. Funding information for this article has been deposited with the Crossref Funder Registry. 


\section{References}

1 Lucas JS, Walker WT, Kuehni CE, et al. Primary ciliary dyskinesia. In: Cordier J-F, ed. Orphan Lung Diseases. Sheffield, European Respiratory Society, 2011; pp. 201-217.

2 Morgan LC, Birman CS. The impact of Primary Ciliary Dyskinesia on the upper respiratory tract. Paediatr Respir Rev 2016; 18: 33-38.

3 Noone PG, Leigh MW, Sannuti A, et al. Primary ciliary dyskinesia: diagnostic and phenotypic features. Am J Respir Crit Care Med 2004; 169: 459-467.

4 Sommer JU, Schafer K, Omran $\mathrm{H}$, et al. ENT manifestations in patients with primary ciliary dyskinesia: prevalence and significance of otorhinolaryngologic co-morbidities. Eur Arch Otorhinolaryngol 2011; 268: 383-388.

5 Morillas HN, Zariwala M, Knowles MR. Genetic causes of bronchiectasis: primary ciliary dyskinesia. Respiration 2007; 74: 252-263.

6 Kennedy MP, Omran H, Leigh MW, et al. Congenital heart disease and other heterotaxic defects in a large cohort of patients with primary ciliary dyskinesia. Circulation 2007; 115: 2814-2821.

7 Shapiro AJ, Davis SD, Ferkol T, et al. Laterality defects other than situs inversus totalis in primary ciliary dyskinesia: insights into situs ambiguus and heterotaxy. Chest 2014; 146: 1176-1186.

8 Hirokawa N, Tanaka Y, Okada Y, et al. Nodal flow and the generation of left-right asymmetry. Cell 2006; 125 $33-45$.

9 Vanaken GJ, Bassinet L, Boon M, et al. Infertility in an adult cohort with primary ciliary dyskinesia: phenotype-gene association. Eur Respir J 2017; 50: 1700314.

10 Halbert SA, Patton D, Zarutskie P, et al. Function and structure of cilia in the fallopian tube of an infertile woman with Kartagener's syndrome. Hum Reprod 1997; 12: 55-58.

11 Lyons RA, Saridogan E, Djahanbakhch O. The reproductive significance of human Fallopian tube cilia. Hum Reprod Update 2006; 12: 363-372.

12 Spassky N. Motile cilia and brain function: ependymal motile cilia development, organization, function and their associated pathologies. In: Tucker K, Caspary T, eds. Cilia and Nervous System Development and Function. Dordrecht, Springer; 2013: pp. 193-207.

13 Halbeisen FS, Jose A, de Jong C, et al. Spirometric indices in primary ciliary dyskinesia: systematic review and meta-analysis. ERJ Open Res 2019; 5: 00231-2018.

14 Hoben IM, Hjeij R, Olbrich $\mathrm{H}$, et al. Mutations in C11orf70 cause primary ciliary dyskinesia with randomization of left/right body asymmetry due to defects of outer and inner dynein arms. Am J Hum Genet 2018; 102 973-984.

15 Kuehni CE, Frischer T, Strippoli MP, et al. Factors influencing age at diagnosis of primary ciliary dyskinesia in European children. Eur Respir J 2010; 36: 1248-1258.

16 O'Callaghan C, Chetcuti P, Moya E. High prevalence of primary ciliary dyskinesia in a British Asian population. Arch Dis Child 2010; 95: 51-52.

17 Onoufriadis A, Paff T, Antony D, et al. Splice-site mutations in the axonemal outer dynein arm docking complex gene CCDC114 cause primary ciliary dyskinesia. Am J Hum Genet 2013; 92: 88-98.

18 Behan L, Dunn Galvin A, Rubbo B, et al. Diagnosing primary ciliary dyskinesia: an international patient perspective. Eur Respir J 2016; 48: 1096-1107.

19 Behan L, Dimitrov BD, Kuehni CE, et al. PICADAR: a diagnostic predictive tool for pirmary ciliary dyskinesia. Eur Respir J 2016; 47: 1103-1112.

20 Mullowney T, Manson D, Kim R, et al. Primary ciliary dyskinesia and neonatal respiratory distress. Pediatrics 2014; 134: 1160-1166.

21 Frija-Masson J, Bassinet L, Honore I, et al. Clinical characteristics, functional respiratory decline and follow-up in adult patients with primary ciliary dyskinesia. Thorax 2017; 72: 154-160.

22 Jackson $\mathrm{AD}$, Goss $\mathrm{CH}$. Epidemiology of $\mathrm{CF}$ : how registries can be used to advance our understanding of the $\mathrm{CF}$ population. J Cyst Fibros 2018; 17: 297-305.

23 Lacaze P, Millis N, Fookes M, et al. Rare disease registries: a call to action. Intern Med J 2017; 47: 1075-1079.

24 Barbato A, Frischer T, Kuehni CE, et al. Primary ciliary dyskinesia: a consensus statement on diagnostic and treatment approaches in children. Eur Respir J 2009; 34: 1264-1276.

25 Farley H, Rubbo B, Bukowy-Bieryllo Z, et al. Proceedings of the 3rd BEAT-PCD Conference and 4th PCD Training School. BMC Proc 2018; 12: 64

26 Lucas JS, Barbato A, Collins SA, et al. European Respiratory Society guidelines for the diagnosis of primary ciliary dyskinesia. Eur Respir J 2017; 49: 1601090.

27 Strippoli MP, Frischer T, Barbato A, et al. Management of primary ciliary dyskinesia in European children recommendations and clinical practice. Eur Respir J 2012; 39: 1482-1491

28 Halbeisen F, Hogg C, Alanin MC, et al. Proceedings of the 2nd BEAT-PCD conference and 3rd PCD training school: part 1. BMC Proc 2018; 12: 1.

29 Rubbo B, Behan L, Dehlink E, et al. Proceedings of the COST action BM1407 inaugural conference BEAT-PCD: translational research in primary ciliary dyskinesia - bench, bedside, and population perspectives. BMC Proc 2016; 10: 66 .

30 Goutaki M, Halbeisen FS, Spycher BD, et al. Growth and nutritional status, and their association with lung function: a study from the international Primary Ciliary Dyskinesia Cohort. Eur Respir J 2017; 50: 1701659.

31 Goutaki M, Maurer E, Halbeisen FS, et al. The international primary ciliary dyskinesia cohort (iPCD Cohort): methods and first results. Eur Respir J 2017; 49: 1601181.

32 Halbeisen FS, Goutaki M, Spycher BD, et al. Lung function in patients with primary ciliary dyskinesia: an iPCD Cohort study. Eur Respir J 2018; 52: 1801040.

33 Halbeisen F, Goutaki M, Maurer E, et al. Lung growth in children and young adults with primary ciliary dyskinesia (PCD): an iPCD cohort study. Eur Respir J 2016; 48: Suppl. 60, PA375.

34 Halbeisen FS, Shoemark A, Barbato A, et al. Time trends in diagnostic testing for PCD in Europe. Eur Respir J 2019; 54: 1900528.

35 Kouis P, Goutaki M, Halbeisen FS, et al. Prevalence and course of disease after lung resection in primary ciliary dyskinesia: a cohort and nested case-control study. Respir Res 2019; 20: 212. 
Werner C, Lablans M, Ataian M, et al. An international registry for primary ciliary dyskinesia. Eur Respir J 2016 47: 849-859.

Alanin MC, Nielsen KG, von Buchwald C, et al. A longitudinal study of lung bacterial pathogens in patients with primary ciliary dyskinesia. Clin Microbiol Infect 2015; 21: 1093.

Green K, Buchvald FF, Marthin JK, et al. Ventilation inhomogeneity in children with primary ciliary dyskinesia. Thorax 2012; 67: 49-53.

Madsen A, Green K, Buchvald F, et al. Aerobic fitness in children and young adults with primary ciliary dyskinesia. PLoS One 2013; 8: e71409.

Maglione M, Bush A, Nielsen KG, et al. Multicenter analysis of body mass index, lung function, and sputum microbiology in primary ciliary dyskinesia. Pediatr Pulmonol 2014; 49: 1243-1250.

Marthin JK, Mortensen J, Pressler T, et al. Pulmonary radioaerosol mucociliary clearance in diagnosis of primary ciliary dyskinesia. Chest 2007; 132: 966-976.

Marthin JK, Nielsen KG. Choice of nasal nitric oxide technique as first-line test for primary ciliary dyskinesia. Eur Respir J 2011; 37: 559-565.

Marthin JK, Nielsen KG. Hand-held tidal breathing nasal nitric oxide measurement - a promising targeted case-finding tool for the diagnosis of primary ciliary dyskinesia. PLoS One 2013; 8: e57262.

Marthin JK, Petersen N, Skovgaard LT, et al. Lung function in patients with primary ciliary dyskinesia: a cross-sectional and 3-decade longitudinal study. Am J Respir Crit Care Med 2010; 181: 1262-1268.

Munkholm M, Nielsen KG, Mortensen J. Clinical value of measurement of pulmonary radioaerosol mucociliary clearance in the work up of primary ciliary dyskinesia. EJNMMI Res 2015; 5: 39.

Alanin MC, Aanaes K, Hoiby N, et al. Sinus surgery can improve quality of life, lung infections, and lung function in patients with primary ciliary dyskinesia. Int Forum Allergy Rhinol 2017; 7: 240-247.

Alanin MC, Johansen HK, Aanaes K, et al. Simultaneous sinus and lung infections in patients with primary ciliary dyskinesia. Acta Otolaryngol 2015; 135: 58-63.

Kobbernagel HE, Green K, Ring AM, et al. One-year evolution and variability in multiple-breath washout indices in children and young adults with primary ciliary dyskinesia. Eur Clin Respir J 2019; 6: 1591841.

Ring AM, Buchvald FF, Holgersen MG, et al. Fitness and lung function in children with primary ciliary dyskinesia and cystic fibrosis. Respir Med 2018; 139: 79-85.

Goutaki M, Halbeisen FS, Wisse AC, et al. Neonatal manifestations in Primary Ciliary Dyskinesia: a multinational cohort study. Eur Respir J 2018; 52: Suppl. 62, PA5021.

Yiallouros PK, Kouis P, Middleton N, et al. Clinical features of primary ciliary dyskinesia in Cyprus with emphasis on lobectomized patients. Respir Med 2015; 109: 347-356.

Yiallouros PK, Kouis P, Pirpa P, et al. Wide phenotypic variability in RSPH9-associated primary ciliary dyskinesia: review of a case-series from Cyprus. J Thorac Dis 2019; 11: 2067-2075.

Goutaki M, Eich MO, Halbeisen FS, et al. The Swiss Primary Ciliary Dyskinesia registry: objectives, methods and first results. Swiss Med Wkly 2019; 149: w20004.

Lucas JS, Burgess A, Mild 2014; 99: 850-856.
Chict

Lucas JS, Chetcuti $\mathrm{P}$, Copeland F, et al. Overcoming challenges in the management of primary ciliary dyskinesia: the UK model. Paediatr Respir Rev 2014; 15: 142-145.

Antony D, Becker-Heck A, Zariwala MA, et al. Mutations in CCDC39 and CCDC40 are the major cause of primary ciliary dyskinesia with axonemal disorganization and absent inner dynein arms. Hum Mutat 2013; 34: 462-472.

Behan L, Leigh MW, Dell SD, et al. Validation of a health-related quality of life instrument for primary ciliary dyskinesia (QOL-PCD). Thorax 2017; 72: 832-839.

Best S, Shoemark A, Rubbo B, et al. Risk factors for situs defects and congenital heart disease in primary ciliary dyskinesia. Thorax 2019; 74: 203-205.

Dell SD, Leigh MW, Lucas JS, et al. Primary ciliary dyskinesia: first health-related quality-of-life measures for pediatric patients. Ann Am Thorac Soc 2016; 13: 1726-1735.

Harris A, Bhullar E, Gove K, et al. Validation of a portable nitric oxide analyzer for screening in primary ciliary dyskinesias. BMC Pulm Med 2014; 14: 18.

Hirst RA, Jackson CL, Coles JL, et al. Culture of primary ciliary dyskinesia epithelial cells at air-liquid interface can alter ciliary phenotype but remains a robust and informative diagnostic aid. PLoS One 2014; 9: e89675.

Jackson CL, Behan L, Collins SA, et al. Accuracy of diagnostic testing in primary ciliary dyskinesia. Eur Respir J 2016; 47: 837-848.

Marino LV, Harris A, Johnstone C, et al. Characterising the nutritional status of children with primary ciliary dyskinesia. Clin Nutr 2019; 38: 2127-2135.

Moore DJ, Onoufriadis A, Shoemark A, et al. Mutations in ZMYND10, a gene essential for proper axonema assembly of inner and outer dynein arms in humans and flies, cause primary ciliary dyskinesia. Am J Hum Genet 2013; 93: 346-356.

Rogers GB, Carroll MP, Zain NMM, et al. Complexity, temporal stability, and clinical correlates of airway bacterial community composition in primary ciliary dyskinesia. J Clin Microbiol 2013; 51: 4029-4035.

Rubbo B, Shoemark A, Jackson CL, et al. Accuracy of high-speed video analysis to diagnose primary ciliary dyskinesia. Chest 2019; 155: 1008-1017.

Shoemark A, Moya E, Hirst RA, et al. High prevalence of CCDC103 p.His154Pro mutation causing primary ciliary dyskinesia disrupts protein oligomerisation and is associated with normal diagnostic investigations. Thorax 2018; 73: 157-166

Strippoli MP, Frischer T, Frischer T, et al. Management of primary ciliary dyskinesia in European children: recommendations and clinical practice. Eur Respir J 2012; 39: 1482-1491.

Tarkar A, Loges NT, Slagle CE, et al. DYX1C1 is required for axonemal dynein assembly and ciliary motility. Nat Genet 2013; 45: 995-1003.

Walker W, Liew A, Harris A, et al. Upper and lower airway nitric oxide levels in primary ciliary dyskinesia, cystic fibrosis and asthma. Respir Med 2013; 107: 380-386.

Walker WT, Jackson CL, Allan RN, et al. Primary ciliary dyskinesia ciliated airway cells show increased susceptibility to Haemophilus influenzae biofilm formation. Eur Respir J 2017; 50: 1700612. 

nitric oxide in response to Haemophilus influenzae infection and proinflammatory cytokines. Chest 2014; 145: 668-669.

73 Walker WT, Young A, Bennett M, et al. Pulmonary radioaerosol mucociliary clearance in primary ciliary dyskinesia. Eur Respir J 2014; 44: 533-535.

74 Irving S, Dixon M, Fassad MR, et al. Primary ciliary dyskinesia due to microtubular defects is associated with worse lung clearance index. Lung 2018; 196: 231-238.

75 Papon J, Coste A, Roudot-Thoraval F, et al. A 20-year experience of electron microscopy in the diagnosis of primary ciliary dyskinesia. Eur Respir J 2010; 35: 1057-1063.

76 Pennarun G, Escudier E, Chapelin C, et al. Loss-of-function mutations in a human gene related to Chlamydomonas reinhardtii dynein IC78 result in primary ciliary dyskinesia. Am J Hum Genet 1999 ; 65 1508-1519.

77 Bequignon E, Dupuy L, Zerah-Lancner F, et al. Critical evaluation of sinonasal disease in 64 adults with primary ciliary dyskinesia. J Clin Med 2019; 8: E619.

78 Fuger M, Aupiais C, Thouvenin G, et al. Gas exchanges in children with cystic fibrosis or primary ciliary dyskinesia: a retrospective study. Respir Physiol Neurobiol 2018; 251: 1-7.

79 Goutaki M, Papon JF, Boon M, et al. Standardised clinical data from patients with primary ciliary dyskinesia (PCD): FOLLOW-PCD. ERJ Open Res 2020; 6: 00237-2019.

80 Goutaki M, Meier AB, Halbeisen FS, et al. Clinical manifestations in primary ciliary dyskinesia: systematic review and meta-analysis. Eur Respir J 2016; 48: 1081-1095.

81 Harris PA, Taylor R, Thielke R, et al. Research electronic data capture (REDCap) - a metadata-driven methodology and workflow process for providing translational research informatics support. J Biomed Inform 2009; 42: 377-381.

82 Videbaek K, Buchvald F, Holgersen MG, et al. The impact of mannose-binding lectin polymorphisms on lung function in primary ciliary dyskinesia. Pediatr Pulmonol 2019; 54: 1182-1189.

83 Robinson P, Morgan L. Bronchiectasis in PCD looks different to CF on CT scan. Multidiscip Respir Med 2018; 13: 24.

84 Tadd K, Morgan L, Rosenow T, et al. CF derived scoring systems do not fully describe the range of structural changes seen on CT scans in PCD. Pediatr Pulmonol 2019; 54: 471-477.

85 Hashimoto N, Hasegawa Y, Keicho N, et al. The nation-wide survey of primary ciliary dyskinesia in Japan. Eur Respir J 2018; 52: Suppl. 62, PA4427.

86 Dasenbrook EC, Sawicki GS. Cystic fibrosis patient registries: a valuable source for clinical research. J Cyst Fibros 2018; 17: 433-440.

87 Viviani L, Zolin A, Mehta A, et al. The European Cystic Fibrosis Society Patient Registry: valuable lessons learned on how to sustain a disease registry. Orphanet J Rare Dis 2014; 9: 81.

88 Chalmers JD, Aliberti S, Polverino E, et al. The EMBARC European Bronchiectasis Registry: protocol for an international observational study. ERJ Open Res 2016; 2: 00081-2015.

89 Guenther A, Krauss E, Tello S, et al. The European IPF registry (eurIPFreg): baseline characteristics and survival of patients with idiopathic pulmonary fibrosis. Respir Res 2018; 19: 141

90 Stockley RA, Luisetti M, Miravitlles M, et al. Ongoing research in Europe: Alpha One International Registry (AIR) objectives and development. Eur Respir J 2007; 29: 582-586.

91 Badesch DB, Raskob GE, Elliott CG, et al. Pulmonary arterial hypertension: baseline characteristics from the REVEAL Registry. Chest 2010; 137: 376-387.

92 Steliarova-Foucher E, Colombet M, Ries LAG, et al. International incidence of childhood cancer, 2001-10: a population-based registry study. Lancet Oncol 2017; 18: 719-731.

93 Pritchard-Jones K, Pieters R, Reaman GH, et al. Sustaining innovation and improvement in the treatment of childhood cancer: lessons from high-income countries. Lancet Oncol 2013; 14: e95-e103.

94 Schindler M, Belle FN, Grotzer MA, et al. Childhood cancer survival in Switzerland (1976-2013): time-trends and predictors. Int J Cancer 2017; 140: 62-74.

95 Bonaventure A, Harewood R, Stiller CA, et al. Worldwide comparison of survival from childhood leukaemia for 1995-2009, by subtype, age, and sex (CONCORD-2): a population-based study of individual data for 89828 children from 198 registries in 53 countries. Lancet Haematol 2017; 4: e202-e217.

96 Spycher BD, Feller M, Röösli M, et al. Childhood cancer and residential exposure to highways: a nationwide cohort study. Eur J Epidemiol 2015; 30: 1263-1275.

97 Spycher BD, Lupatsch JE, Zwahlen M, et al. Background ionizing radiation and the risk of childhood cancer: a census-based nationwide cohort study. Environ Health Perspect 2015; 123: 622-628.

98 Bhatia S, Armenian SH, Armstrong GT, et al. Collaborative research in childhood cancer survivorship: the current landscape. J Clin Oncol 2015; 33: 3055-3064.

99 Forrest CB, Margolis PA, Bailey LC, et al. PEDSnet: a national pediatric learning health system. J Am Med Inform Assoc 2014; 21: 602-606.

100 Wi C-I, Sohn S, Rolfes MC, et al. Application of a natural language processing algorithm to asthma ascertainment. An automated chart review. Am J Respir Crit Care Med 2017; 196: 430-437. 